\title{
Effect of transplantation of marrow mesenchymal stem cells transfected with insulin-like growth factor-1 gene on fracture healing of rats with diabetes
}

\author{
Xing $\mathrm{DG}^{1}$, Liu $\mathrm{ZH}^{2}$, Gao $\mathrm{HW}^{2}$, $\mathrm{Ma} \mathrm{WL}^{2}$, Nie L'르. Gong $\mathrm{MZ}^{2}$ \\ Department of Orthopaedics, the Qilu Hospital of Shandong University, Jinan, People's Republic of China. \\ 56881066@qq.com
}

\begin{abstract}
Objective: To observe the effect of transplantation of marrow mesenchymal stem cells (BMSCs) transfected with insulin-like growth factor-1 (IGF-1) on fracture healing of rats with diabetes and discuss the gene therapy for diabetic fracture.

Methods: 60 8-week-old male Wistar rats weighing 180-200 g were divided into the control group and experimental group at random. All of them suffered from right tibia fracture following the model of diabetes induced by streptozotocin. BMSCs were transfected with Ad- IGF-1 and BMSCs of the appropriate group were transplanted to part of the fracture area. 6 rats were selected from each group at 1, 2, 3, 5 and 7 weeks after the surgery. Local bone callus was stained with hematoxylin-eosin (H-E) and IGF-1 in the bone callus and serum was tested. Results: Osteoid tissues formed at 3 weeks in the experimental group; mature lamellar bone formed at 7 weeks in the experimental group; fibrous bone callus was observed in the control group. IGF-1 in bone callus of the experimental group is increasing and significantly different from that of the control group $(p<0.05)$. Concentrations of IGF-1 in the serum of the two groups were increasing gradually from the first week. The control group reached its peak in the 5th week. The experimental group reached a high concentration in the 5th week and maintained a high concentration in the 7th week. The differences at various times between the two groups have statistical significance $(p<0.05)$.

Conclusion: Transplantation of BMSCs transfected with IGF-1 gene can promote fracture healing of rats with diabetes (Tab. 4, Fig. 1, Ref. 20). Text in PDF www.elis.sk.

Key words: insulin-like growth factor-1, marrow mesenchymal stem cells, diabetes, fracture, healing, rats.
\end{abstract}

Fracture healing includes regulation on differentiation and proliferation of marrow mesenchymal stem cells by cytokines and cytokine osteoinduction. Bone morphogenetic protein-2 (BMP-2) and insulin-like growth factor-1 (IGF-1) are important cytokines influencing fracture healing (1). BMP-2 can induce marrow mesenchymal stem cells (BMSCs) to differentiate into osteocytes and is the most powerful factor in osteogenesis. IGF-1 can promote formation and differentiation osteoblasts and has a function similar to insulin (2-3). Diabetic fracture healing is lower than that of a normal organism. Researchers have demonstrated that during fracture healing of rats with diabetic fracture, the osteocalcin level is low, the capability of osteoblasts transformation weakens and fracture healing slows down (4-5). How to promote healing of the organism with diabetic fracture is a problem requiring urgent solution. We transplanted the marrow mesenchymal stem cells (BMSCs) transfected with insulin-like growth factor-1 (IGF1) gene in the in vitro high glucose environment into part of the diabetic fracture area and observed its effect on fracture healing.

${ }^{1}$ Department of Orthopaedics, the Qilu Hospital of Shandong University, Jinan, People's Republic of China, and ${ }^{2}$ Department of Orthopaedics, the Second Hospital of Shandong University, Jinan, People's Republic of China Address for correspondence: L. Nie, Department of Orthopaedics, The Qilu Hospital of Shandong University, Wenhua Xi Street No. 107, Shandong 250012, Jinan, People's Republic of China

\section{Material and methods}

\section{Experimental material}

60 8-week-old male Wistar rats weighing 180-200g, purchased from SHANGHAI SLAC LABORATORY ANIMAL CO. LTD., Certificate of Quality No. SCXK(Shanghai)2013-0008.

Streptozotocin (STZ) was purchased from Beijing Ruixiang Biotechnology Co., Ltd. Rats BMSCs were purchased from Shanghai Boyan Biotechnology Co., Ltd. Ad-IGF-1 were purchased from Shanghai Weijing Biotechnology Co., Ltd. Trypsin and high glucose DMEM medium were purchased from China Diagnostics Medical Corporation. SABC immunologic tissue chemical reagent kit and ED1022 were purchased from U.S. Sigma Company. Rat enzyme-linked immunoassay adsorption test (ELISA) reagent kits were purchased from U.S. Fermentas Company.

\section{Culture and gene transfection of rat BMSCs}

Primary rat BMSCs were inoculated to the special medium containing FBS with $10 \%$ volume fraction after recovery, at $37^{\circ} \mathrm{C}$, under saturation humidity, and placed into an incubator containing $\mathrm{CO}_{2}$ with $5 \%$ volume fraction for culture. The solution was replaced after $24 \mathrm{~h}$ and non-adherent cells were removed. The solution was replaced once every $2 \mathrm{~d}$ afterwards. Pancreatic enzymes and EDTA were used for dissociation after cells reached $90 \%$ 
confluency. Passage was performed as per $1: 3$ until confluence of the 3 rd-generation cells. The medium were pipetted and $2 \mathrm{ml}$ virus working solution of appropriate group was added to each vessel. Transfection was performed for virus with 100 multiplicity of infection at $37^{\circ} \mathrm{C}$ overnight. It was placed in the high glucose medium for continuous culture.

Testing expression of target gene after cell transfection with RTPCR method

RNA of transfected BMSCs of Wistar rats in the high glucose environment was extracted at $24 \mathrm{~h}, 38 \mathrm{~h}, 72 \mathrm{~h}$ and $96 \mathrm{~h}$ in accordance with the steps of cell lysis, phase separation, RNA sediment, RNA washing, RNA redissolution, RNA quality assessment, RNA purity and yield assessment and stored at $-20{ }^{\circ} \mathrm{C}$. Then purification was performed for RNA. Inverse transcription was performed for the purified RNA sample. Finally, real-time polymerase chain reaction (RT-PCR) test was conducted and data collection and reduction were performed.

Preparation of diabetic fracture model and stem cell transplantation

60 male Wistar rats were divided into the control group and the experimental group at random with 30 for each group. Rats of each group abstained from food for $24 \mathrm{~h}$. They were intraperitoneally injected with STZ as per $30 \mathrm{mg} / \mathrm{kg}$ body mass and raised with ordinary feed for $7 \mathrm{~d}$. Caudal vein blood was collected to test the fasting and random blood glucose. Blood glucose $>11.2 \mathrm{mmol} / \mathrm{L}$ indicated that diabetes model was successfully induced. Rats of each group underwent general anesthesia. An incision was made aseptically to expose the superior segment of tibia. The tibia sawedoff approximately $1 \mathrm{~cm}$ below the tibial plateau. The incision was sutured and fixed with a splint after reduction. BMSCs suspension of the appropriate group was injected into the vicinity of the fracture area through the normal skin with an injector for medical purpose. Local compression was performed for $10 \mathrm{~min}$. The number of stem cells transplanted for each rat is $1 \times 10^{6}$. BMSCs undergoing no gene transfection were transplanted for the control group and BMSCs transfected with Ad- IGF-1 were transplanted for the experimental group.

\section{Specimen collection}

6 rats were selected from each group at 1, 2, 3, 5, 7 week (s) after operation. $5 \mathrm{ml}$ blood was collected from the portal vein and centrifuged at $3000 \mathrm{r} / \mathrm{min}$ for $10 \mathrm{~min}$. The supernatant was decanted and stored at $-20{ }^{\circ} \mathrm{C}$ for test. The bone callus tissues were put into the formaldehyde with volume fraction of $3 \%$ and fixed for $24 \mathrm{~h}$. Then they were decalcified at $4{ }^{\circ} \mathrm{C}$ for 3 weeks by $100 \mathrm{~g} / \mathrm{L}$ edetic acid (EDTA) with $\mathrm{pH}$ value of 7.2. Decalcification terminated until the cortical bone could be penetrated by a needle easily. Gradient ethanol dehydration and paraffin embedding were performed. They were stained with hematoxylin-eosin (H-E) after being sectioned.

\section{Immunohistochemical detection of IGF-1 in bone callus}

The sections were stained with the SABC method and developed with DBA. The specific operating steps should in accordance with the instructions. The average gray value was analysed by the Leica Qwin V3 image analyzer. The lower the gray value, the higher the IGF-1 expression.

\section{Detection of IGF-1 in serum with the ELISA method}

The specific method should be in accordance with the instructions. The absorbance value (A) of each well was measured at a wavelength of $450 \mathrm{~nm}$ after the reaction ceased. The regression equation of the standard curve was calculated and the sample concentration was calculated through the equation based on A value of the sample.

\section{Statistical methods}

The SPSS 15.0 software was used for statistical analysis. The data was expressed with the mean \pm standard deviation $( \pm \mathrm{s})$. The $\mathrm{t}$ test was conducted for comparison among the groups.

\section{Results}

Experimental result of reverse transcription-polymerase chain reaction ( $R T-P C R)$

The expression quantity of target genes in cells exhibits an increasing trend after a successful target gene transfection. IGF-1 at difference times was statically different from that of the control group $(\mathrm{p}<0.05)$ (Tab. 1).

\section{Detection of IGF-1 concentration after cell transfection with ELISA}

The concentration of the product increases after successful target gene transfection. IGF-1 at different times was statistically different from the control group $(\mathrm{p}<0.05)$ (Tab. 2).

\section{HE staining result}

Osteoid tissues formed and a number of chondrocytes and osteoblasts formed in the experimental group 3 weeks after HE staining. Mature lamellar bone formed at 7 weeks in the experimental group; fibrous bone callus was observed in the control group (Fig. 1).

\section{Expression of IGF-1 in bone callus}

IGF-1 exhibited a positive expression in osteoblasts, chondroblasts and marrow mesenchymal stem cells in the experimental

Tab. 1. IGF-1 Gray values at different times.

\begin{tabular}{lccc}
\hline Group & \multicolumn{3}{c}{ Time from Target Gene Transfection (h) } \\
\cline { 2 - 4 } & 24 & 48 & 72 \\
\hline Control group & $0.63 \pm 0.11$ & $0.66 \pm 0.12$ & $0.67 \pm 0.10$ \\
Experimental group & $1.15 \pm 0.14^{*}$ & $1.22 \pm 0.16^{*}$ & $0.64 \pm 0.12$ \\
\hline
\end{tabular}

Compared with the control group, $* \mathrm{p}<0.05$ 

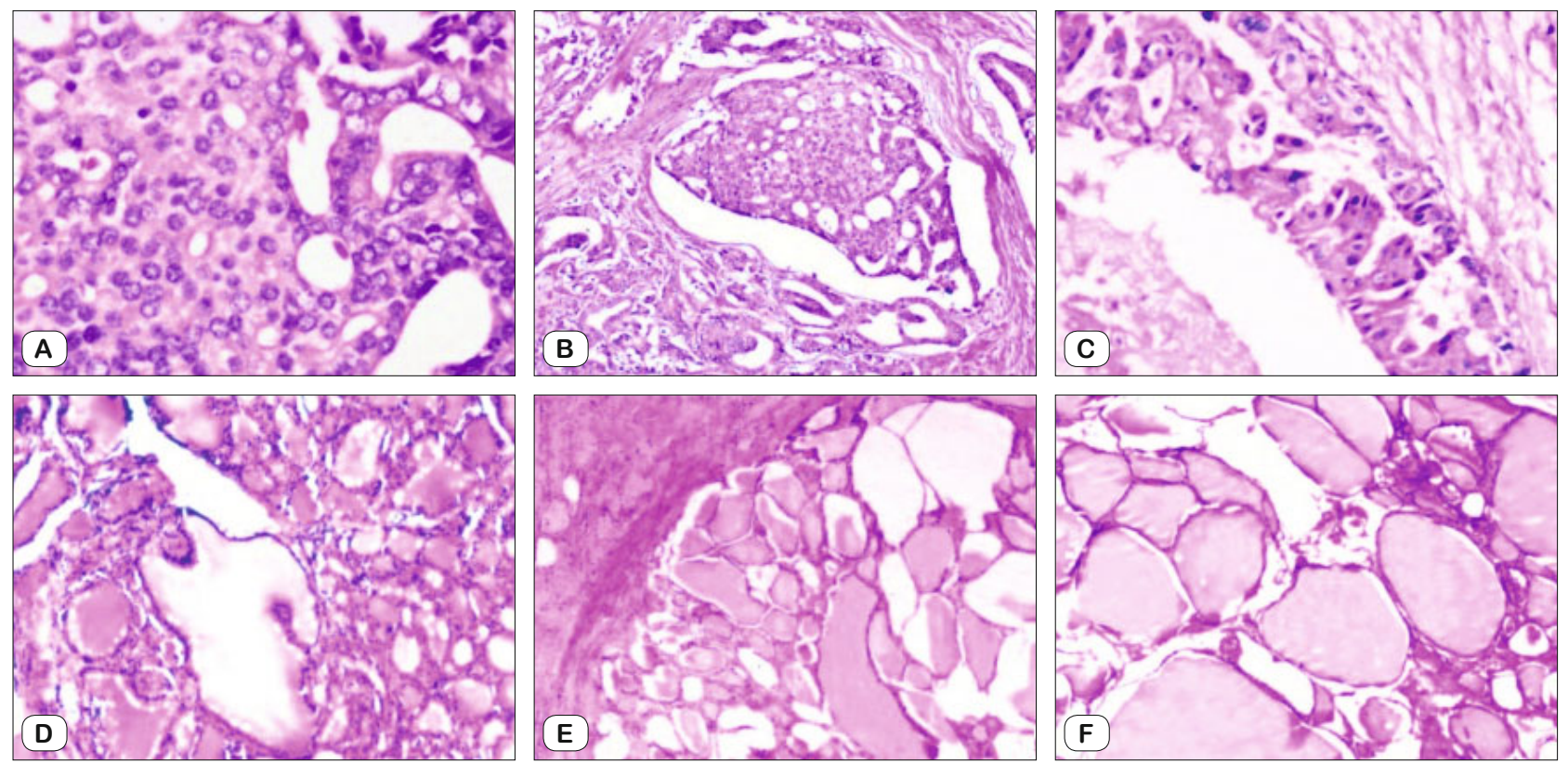

Fig. 1. HE staining results for bone callus tissues of rats at different times. A - the control group; B - 1 week after stem cell transplantation; C - 2 weeks after stem cell transplantation; D - 3 weeks after stem cell transplantation; E - 5 weeks after stem cell transplantation; F - 7 weeks after stem cell transplantation.

group and a weak positive expression in fewer osteoblasts, mesenchymal stem cells, fibroblasts and chondroblasts in the control group in the first week. IGF-1 exhibited a positive expression in osteoblasts, mesenchymal stem cells, chondrocytes and bone matrix in the experimental group and a little expression in osteoblasts, mesenchymal stem cells and chondrocytes in the control group in the second week, which were enhanced compared to the first week. IGF-1 expression reached its peak in marrow mesenchymal stem cells, osteoblasts, new bone matrix and chondrocytes in the control group in the 5th week but was still weak compared to the experimental group. Expression of IGF-1 in marrow mesenchymal stem cells, osteoblasts and chondrocytes increased continuously in the experimental group in the 7th week and expression in the control group weakened. Upon processing by the Leica Qwin V3 image analysis system, IGF-1 gray value of the experimental group was lower than that of the control group and such a difference had a statistical significance $(\mathrm{p}<0.05)$ (Tab. 3).

\section{Concentration of IGF-1 in serum}

Concentrations of IGF-1 in the serum of the two groups were increasing gradually from the first week. The control group reached its peak in the 5 th week. The experimental group reached a high

Tab. 2. IGF-1 protein product concentration at different times (ng/L).

\begin{tabular}{lccc}
\hline Group & \multicolumn{3}{c}{ Time from Target Gene Transfection (h) } \\
\cline { 2 - 4 } & 24 & 48 & 72 \\
\hline Control group & $32.47 \pm 1.68$ & $33.04 \pm 1.57$ & $34.71 \pm 1.62$ \\
Experimental group & $48.66 \pm 2.17^{*}$ & $53.12 \pm 2.30^{*}$ & $33.82 \pm 1.55$ \\
\hline Compared & $65.24 \pm 2.43^{*}$ & $60.16 \pm 2.41^{*}$ \\
\hline
\end{tabular}

Compared with the control group, $* \mathrm{p}<0.05$

Tab. 3. IGF-1 Gray values of the two groups of rats at different times.

\begin{tabular}{|c|c|c|c|c|c|}
\hline \multirow[t]{2}{*}{ Group } & \multicolumn{5}{|c|}{ Time from transplantation (week) } \\
\hline & 1 & 2 & 3 & 5 & 7 \\
\hline Control group & $86.34 \pm 3.65$ & $80.27 \pm 3.44$ & $77.68 \pm 3.35$ & $71.15 \pm 3.31$ & $62.98 \pm 3.27$ \\
\hline Experimental group & $65.92 \pm 2.97 *$ & $58.16 \pm 2.76^{*}$ & $51.28 \pm 2.49 *$ & $42.16 \pm 2.42 *$ & $30.68 \pm 2.24 *$ \\
\hline
\end{tabular}

Compared with the control group, $* \mathrm{p}<0.05$

Tab. 4. Concentration of IGF-1 in bone callus of the two groups of rats at different times (ng/L).

\begin{tabular}{|c|c|c|c|c|c|}
\hline \multirow[t]{2}{*}{ Group } & \multicolumn{5}{|c|}{ Time from transplantation (week) } \\
\hline & 1 & 2 & 3 & 5 & 7 \\
\hline Control group & $338.96 \pm 26.51$ & $357.68 \pm 27.13$ & $365.82 \pm 27.46$ & $382.17 \pm 28.34$ & $372.62 \pm 27.69$ \\
\hline Experimental group & $376.57 \pm 25.94 *$ & $406.92 \pm 27.67 *$ & $426.85 \pm 28.47 *$ & $449.28 \pm 28.19 *$ & $468.37 \pm 29.16^{*}$ \\
\hline
\end{tabular}

Compared with the control group, $* \mathrm{p}<0.05$ 
concentration in the 5 th week and maintained a high concentration in the 7 th week. The differences at various times between the two groups had a statistical significance $(\mathrm{p}<0.05)$ (Tab. 2).

\section{Discussion}

Fracture healing is a complex process participated in by multiple cells and cytokines. BMSCs are seed cells, which play an important role in the process. They can differentiate into osteoblasts and chondrocytes. They are the ideal target cells for gene transfection. Currently, multiple types of bone cytokines, among which IGF-1 is the cytokine rich in content in osteocytes, which promotes composition and mineralization of bone matrix and plays an important role in fracture healing (8-9). Some researchers have demonstrated that BMSCs can be successfully transfected by IGF-1 gene in a high glucose environment. Gene transfection can promote BMSCs proliferation and differentiation. Transfected BMSCs can constantly exhibit a high expression level (10-11). The research results showed that IGF-1 in serum and bone callus of rats with diabetic fracture decreases and fracture healing delayed relatively. It can be inferred that decreasing IGF-1 is one of the reasons for delayed diabetic fracture. We transplanted BMSCs transfected by IGF-1 gene in a high glucose environment into part of fracture area of rats with diabetic fracture to discuss the relation between IGF-1 expression and fracture healing. The results showed that IGF-1 exhibited a constant high expression in rats with diabetes accelerating fracture healing to some extent, which will provide an experimental basis for clinical probe into the new gene therapy for diabetic fracture.

The research results showed that contents of IGF-1 in bone callus and serum increase after BMSCs transfected with IGF-1 gene in in-vitro high glucose environment. IGF-1 expression in the experimental group and the control group increased in the first five weeks whereas IGF-1 expression in the experimental group significantly increased compared to the control group. It was considered that such results mainly related to IGF-1's role in promoting proliferation after gene transfection and IGF-1 promoting BMSCs to differentiate into osteoblasts and chondroblasts. The differentiated osteoblasts, chondroblasts and bone matrix further express IGF-1 leading to positive stimulation of IGF-1 on BMSCs and osteoblasts and constant high expression of IGF-1 (12-13). Increased IGF-1 will influence fracture healing by autocrine, paracrine and endocrine (14-15). Reistetter et al have demonstrated in their researches that expressions of IGF-1 of rats with diabetes and its receptor decreased. Some researchers also have demonstrated that osteoblasts differentiation of patients with diabetes was inhibited, number of osteoblasts decreased and bone turnover cycle extended accordingly due to the fact that patients with diabetes were affected by such chronic factors as metabolism (16). It is thus clear that diabetes is a negative factor for fracture healing and decreasing IGF-1 expression plays a vital role. Norris and other have demonstrated that IGF-1 could act on osteoblasts to accelerate proliferation and differentiation of osteoblasts and promote osteogenesis (17). The research results showed that expression of IGF-1 in bone callus and serum of rats with diabetic fracture increases. IGF-1 with a higher concentration acts on BMSCs and osteoblasts through the above approach and promotes proliferation and differentiation and bone matrix synthesis. Hence, it can reverse the negative effect of diabetes on fracture healing and accelerate fracture healing of rats with diabetes to some extent. In the 7th week, after the fracture basically healed, expression level of IGF-1 in the experimental group still remained high due to simultaneous osteogenesis and bone resorption. A great deal of IGF-1 released when bone resorption occurred and IGF-1 had very strong osteogenesis. At the moment, IGF-1 mediated balance between osteogenesis and bone resorption promoted formation of mature lamellar bone and helped bone remodeling.

Some researchers have demonstrated that BMSCS can improve biomechanical properties of bone callus tissues, increase cartilage and bone mass during bone repair and play a vital role in initiation of fracture healing and bone repair (18). BMSCS transplanted in the experiment increased the number of BMSCS in rats with diabetes. Fracture healing accelerated in the experimental group when the numbers of BMSCS increased in the experimental group and the control group were identical indicating an increased expression of IGF-1 after gene transfection played a vital role in fracture healing in addition to BMSCS. The experiment enabled IGF-1 to be highly and constantly expressed in vivo through the techniques of gene transfection and stem cells, discussed the new method to promote diabetic fracture healing, overcame the disadvantages in application of exogenous cytokines, such as high cost, poor controllability, short half-life period etc. However, fracture healing is a process where multiple cells and factors participate. Diabetic fracture healing is more complex and a conundrum requiring urgent solution (19-20).

The followings still remain unclear and need further researches: specific differentiation and action mechanism after BMSCS transfected with gene were transplanted into the body, whether BMSCS transfected simultaneously with multiple genes can be transplanted into the diabetic organism for treatment of diabetic fracture and how to safely apply such a technique to clinical treatment of patients with diabetic fracture etc.

\section{References}

1. Paglia DN, Wey A et al. Effects of local insulin delivery on subperiosteal angiogenesis and mineralized tissue formation during fracture healing. J Orthop Res 2013; 31 (5): 783-791.

2. Leslie WD, Aubry-Rozier B et al. TBS (trabecular bone score) and diabetes-related fracture risk. J Clin Endocrinol Metab 2013; 98 (2): 602-609.

3. Allison SJ. Transplantation: Pancreas-kidney transplantation may reduce fracture risk in men with type 1 diabetes mellitus. Nat Rev Nephrol 2013; 9 (3): 124-127.

4. Nirantharakumar K, Toulis KA et al. Impact of diabetes on inpatient mortality and length of stay for elderly patients presenting with fracture of the proximal femur. J Diabetes Complications 2013; 27 (3): 208-210.

5. Nan HL, Zhang Y et al. A case of Klinefelter's syndrome with refractory asthma, diabetes mellitus and rib fracture. Chin Med J (Engl) 2013; 126 (1): 196-198.

\section{FAILED !!!}


64-68

7. Ekstrom W, Al-Ani AN et al. Health related quality of life, reoperation rate and function in patients with diabetes mellitus and hip fracture-A 2 year follow-up study. Injury 2013; 44 (6): 769-775.

8. Takaoka S, Yamaguchi T et al. Fracture risk is increased by the complication of hypertension and treatment with calcium channel blockers in postmenopausal women with type 2 diabetes. J Bone Miner Metab 2013; 31 (1): 102-107.

9. Huang YF, Shyu YI et al. Diabetes and health outcomes among older Taiwanese with hip fracture. Rejuvenation Res 2012; 15 (5): 476-482.

10. Simpson CM, Calori GM et al. Diabetes and fracture healing: the skeletal effects of diabetic drugs. Expert Opin Drug Saf 2012; 11 (2): 215-220.

11. Giangregorio LM, Leslie WD et al. FRAX underestimates fracture risk in patients with diabetes. J Bone Miner Res 2012; 2 7(2): 301-308.

12. Fraser $\mathbf{L A}$, Pritchard $\mathbf{J}$ et al. Clinical risk factors for fracture in diabetes: a matched cohort analysis. J Clin Densitom 2011; 14( 4): 416-421.

13. Yamaguchi T, Sugimoto $\mathbf{T}$ et al. Bone metabolism and fracture risk in type 2 diabetes mellitus (Review). Endocr J 2011; 58 (8): 613-624.
14. Montagnani A, Gonnelli S et al. Osteoporosis and risk of fracture in patients with diabetes: an update. Aging Clin Exp Res 2011; 23 (2): 84-90.

15. Betteridge DJ. Thiazolidinediones and fracture risk in patients with Type 2 diabetes. Diabet Med 2011; 28 (7): 759-771.

16. Reistetter TA Graham JE et al. Diabetes comorbidity and age influence rehabilitation outcomes after hip fracture. Diabetes Care 2011; 34 (6): 1375-1377.

17. Norris R, Parker M. Diabetes mellitus and hip fracture: a study of 5966 cases. Injury 2011; 42 (11): 1313-1316.

18. Tatsuno I, Sugiyama T. Glucocorticoid-induced diabetes mellitus is a risk for vertebral fracture during glucocorticoid treatment. Diabetes Res Clin Pract 2011; 93 (1): 18-20.

19. Shan PF, Wu XP et al. Age-related bone mineral density, osteoporosis rate and risk of vertebral fracture in mainland Chinese women with type 2 diabetes mellitus. J Endocrinol Invest 2011; 34 (3): 190-196.

20. Schafer AL, Vittinghoff $\mathbf{E}$ et al. Fat infiltration of muscle, diabetes, and clinical fracture risk in older adults. J Clin Endocrinol Metab 2010; 95 (11): 368-372. 